\title{
Influencia del paisaje en las cortas de protección en bosques de Nothofagus pumilio en Tierra del Fuego, Argentina: Cambios en la estructura forestal y respuesta de la regeneración
}

\author{
Landscape influence in the shelterwood cuts of Nothofagus pumilio forests in Tierra del Fuego, \\ Argentina: Changes in forest structure and regeneration performance
}

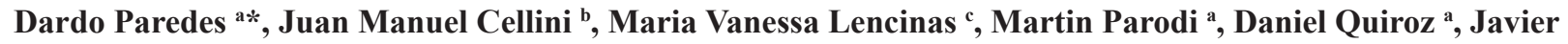 \\ Ojeda $^{a}$, Sebastian Farina a, Yamina Micaela Rosas c, Guillermo Martínez Pastur c \\ *Autor de correspondencia: a Dirección General de Desarrollo Forestal (DGDF), \\ Ministerio de Producción y Ambiente (MPyA) del Gobierno de Tierra del Fuego, Angela Loig 211 (9412) Tolhuin, \\ Tierra del Fuego, Argentina, dparedes@tierradelfuego.gov.ar \\ ${ }^{\mathrm{b}}$ Laboratorio de Investigaciones en Madera (LIMAD), Universidad Nacional de La Plata (UNLP), \\ Diagonal $113 n^{\circ} 469$ (1900) La Plata, Buenos Aires, Argentina. \\ ${ }^{\mathrm{c}}$ Centro Austral de Investigaciones Científicas (CADIC), Consejo Nacional de Investigaciones Científicas y Técnicas (CONICET), \\ Houssay 200 (9410) Ushuaia, Tierra del Fuego, Argentina.
}

\section{SUMMARY}

During the last thirty years, shelterwood cuts has been the most applied regeneration method in Nothofagus pumilio (lenga) forests. This treatment was implemented without any consideration of the landscape, and without considering yield gradients (e.g. stand site qualities), environmental restrictions (e.g. soil water availability) or potential natural disturbance factors (e.g. wind exposure). The objective was to evaluate the dynamics of the remnant forest structure and the natural regeneration 10 years after harvesting across the landscape considering different environmental gradients. Sampling plots were established in nine stands distributed across Tierra del Fuego, Argentina (north, east and south areas) where forestry activity has been concentrated during the last years. The selected areas presented differences in the rainfall regime (402 to $446 \mathrm{~mm} \mathrm{yr}^{-1}$ ) and site qualities of the stands (2.6 to 3.3). Forest structure was similar among the studied areas, nevertheless harvesting intensity was variable (30\% to $57 \%$ ) generating different remnant basal areas (BA) $\left(22\right.$ to $\left.47 \mathrm{~m}^{2} \mathrm{ha}^{-1}\right)$ and woody debris accumulation in the forest floor $\left(167\right.$ to $\left.230 \mathrm{~m}^{3} \mathrm{ha}^{-1}\right)$ after harvesting, or in combination with local wind-throws. The current forest structure presented differences in BA (6 to $\left.34 \mathrm{~m}^{2} \mathrm{ha}^{-1}\right)$ and established regeneration (49 to 110 thousand $\left.\mathrm{ha}^{-1}\right)$ that varied in height $(0.6$ to $1.0 \mathrm{~m})$ and growth $\left(4.1\right.$ to $\left.12.8 \mathrm{~cm} \mathrm{yr}^{-1}\right)$ according to the area and the received damage due to abiotic and biotic factors. We can conclude that forests located along the considered landscapes and environmental gradients differentially responded to the proposed silvicultural treatments, where climate influenced over the post-harvest canopy stability and the established regeneration. For this, it is mandatory to define different silvicultural strategies throughout the landscape.

Key words: forest dynamics, remnant forest canopy, natural regeneration, browsing, abiotic damage.

\section{RESUMEN}

El método de regeneración más aplicado en bosques de Nothofagus pumilio (lenga) es la corta de protección. Este tratamiento se implementó sin consideración del paisaje o gradientes de productividad (ej. calidad de sitio), restricciones ambientales (ej. humedad del suelo) o factores de disturbio (ej. viento). El objetivo fue evaluar la dinámica de la estructura forestal remanente y la respuesta de la regeneración luego de 10 años de cosecha en el paisaje considerando gradientes ambientales. Se establecieron parcelas en nueve rodales en distintos sectores de Tierra del Fuego, Argentina (norte, este y sur) donde se concentra la actividad forestal. Las zonas seleccionadas presentan diferencias en el régimen hídrico (402 a $446 \mathrm{~mm}$ año-1) y calidades de sitio (2,6 a 3,3). La estructura forestal fue similar entre zonas, pero las intensidades de corta fue variable (30\% a 57\%) generando diferentes áreas basales (AB) remanentes (22 a $47 \mathrm{~m}^{2} \mathrm{ha}^{-1}$ ) y residuos forestales (167 a $230 \mathrm{~m}^{3} \mathrm{ha}^{-1}$ ) luego de la cosecha o por volteos de viento. La estructura forestal actual presenta diferencias en $\mathrm{AB}\left(6\right.$ a $\left.34 \mathrm{~m}^{2} \mathrm{ha}^{-1}\right)$ y regeneración instalada (49 a $\left.110 \mathrm{miles} \mathrm{ha}^{-1}\right)$ variando en altura $(0,6$ a $1,0 \mathrm{~m})$ y crecimiento $\left(4,1\right.$ a $\left.12,8 \mathrm{~cm}_{\text {año }}{ }^{-1}\right)$ por zona y daños recibidos (abióticos y bióticos). Concluimos que los bosques responden diferencialmente a los tratamientos silvícola en los paisajes y gradientes ambientales analizados, donde el clima influye en la estabilidad del dosel y la regeneración. En consecuencia, se hace necesario establecer estrategias silvícolas diferenciales a lo largo del paisaje.

Palabras clave: dinámica forestal, dosel remanente, regeneración natural, ramoneo, daños abióticos. 


\section{INTRODUCCIÓN}

La Isla Grande de Tierra del Fuego en Argentina reúne variadas condiciones fisiográficas y climáticas que se reflejan en su fisionomía vegetal, pudiéndose dividir la misma en cuatro regiones naturales: estepa magallánica, ecotono estepa-bosque, cordillera al oeste y humedales al este (Allué et al. 2010). Los bosques de Nothofagus pumilio (Poepp. et Endl.) Krasser (comúnmente llamada lenga) son los que poseen la mejor aptitud maderera con destino al aserrado (Martínez Pastur et al. 2009). Se encuentran distribuidos en la zona centro-sur de la Isla, ocupando las áreas desde el ecotono estepa-bosque hasta la cordillera, donde se mezclan con los bosques mixtos siempreverdes hacia la región de los humedales al oeste.

Los sistemas silvícolas utilizados en los bosques de lenga han cambiado a lo largo del tiempo, desde cortas selectivas hasta talas rasas (López Bernal et al. 2003, Gea et al. 2004), variando las intensidades de corta de acuerdo a los productos extraídos. El sistema de regeneración más aplicado a finales del siglo pasado, tanto en Chile como en Argentina, fue el de cortas de protección, así como otras alternativas que combinan diferentes niveles de retención (Rosenfeld et al. 2006, Martínez Pastur et al. 2009) que consisten en mantener elementos estructurales del bosque original que satisfacen requerimientos de hábitat para ciertos organismos (ej. retención dispersa y/o retención agregada). La corta de protección en particular consiste en la intervención progresiva del bosque, que se inicia con una primera corta que abre el dosel dejando $30 \mathrm{~m}^{2} \mathrm{ha}^{-1}$ de área basal (AB) con una distancia máxima entre árboles de $12 \mathrm{~m}$ uniformemente distribuidos, a los fines de estimular el establecimiento y crecimiento de la regeneración natural (Martínez Pastur et al. 2007, Cellini et al. 2013). Estas modificaciones en el dosel del bosque impactan fuertemente sobre las condiciones microclimáticas, generando un aumento de la temperatura del suelo, la precipitación efectiva, la intensidad lumínica y la velocidad del viento dentro del bosque (Martínez Pastur et al. 2011, 2013, Soto et al. 2017, 2019), causando un incremento en la evapotranspiración e influyendo en la dinámica y crecimiento de la regeneración, tanto pre- como post-instalada respecto del momento de la cosecha (Rosenfeld et al. 2006, Martínez Pastur et al. 2007, 2017, Pérez Flores et al. 2019).

El período de regeneración natural se extiende por un largo tiempo (15 a 20 años), donde ocurren daños en el dosel remanente debido al rastreo durante la cosecha o por efecto del viento al abrir el dosel (Cellini et al. 2013). La regeneración establecida bajo este dosel de protección genera un denso bosque secundario, y es entonces cuando se realizan las siguientes intervenciones (otras cortas intermedias) para seguir abriendo el dosel o cortas finales en caso que el objetivo del manejo sea transformar totalmente el bosque primario en bosques manejados de alta productividad (Gea et al. 2004, Cellini et al. 2013, Martínez
Pastur et al. 2013). La regeneración natural puede verse afectada tanto por factores bióticos (ej. herbivoría) como abióticos (ej. heladas y sequías) (Martínez Pastur et al. 2013, 2016, 2017). La herbivoría abarca un amplio espectro de posibilidades, desde la predación de frutos (ej. aves, insectos y roedores) hasta las plantas establecidas en el sotobosque que son consumidas por diversos mamíferos nativos (ej. el guanaco, Lama guanicoe Müller) o introducidos (ej. el castor, Castor canadensis Kuhl) incluyendo al ganado doméstico, tanto el que está bajo manejo como el ganado vacuno y caballar en estado salvaje. La herbivoría sobre la regeneración natural genera una presión continua, pero que, en general, no llega a poner en peligro la recuperación del bosque a escala de rodal (Gea et al. 2004, Cavieres y Fajardo, 2005, Martínez Pastur et al. 2013, 2017). Sin embargo, el ramoneo continuado supone una detención del crecimiento que puede extenderse en el tiempo en la medida en que perduren las condiciones de elevada presión sobre las plantas afectadas, generalmente a una escala puntual dentro del paisaje (Martínez Pastur et al. 2016). Al igual que la herbivoría, los factores abióticos afectan parte de la biomasa aérea de la regeneración, pudiendo generar la muerte parcial o total de la biomasa aérea frente a eventos extremos o afecciones sucesivas (Martínez Pastur et al. 2017).

El principal inconveniente en la implementación de las cortas de protección en Tierra del Fuego, desde finales del siglo pasado hasta el presente, se debe a que se ha realizado sin consideración alguna del paisaje (ecotono estepa-bosque, bosques de cordillera, o bosques húmedos), y sin tener en cuenta gradientes de productividad (ej. calidad de sitio), potenciales restricciones ambientales (ej. disponibilidad de agua en el suelo) o potenciales factores de disturbio en masa (ej. exposición al viento). El objetivo de este trabajo fue estudiar la influencia del paisaje a lo largo de gradientes ambientales en la implementación de cortas de protección en bosques de lenga, analizando la dinámica de la estructura forestal remanente y la respuesta de la regeneración natural luego de 10 años de realizada la cosecha. Con esta investigación se pretende responder las siguientes preguntas respecto de los gradientes ambientales: (i) ¿varía la estructura forestal de los bosques sin manejo a lo largo de estos gradientes?; (ii) ¿la intensidad de cosecha implementada fue similar a lo largo de estos gradientes?; (iii) ¿varió la dinámica del dosel remanente en la última década a lo largo de estos gradientes?; (iv) ¿cómo fue la respuesta de la regeneración a la cosecha a lo largo de estos gradientes?, y (v) ¿fueron similares los daños observados en la regeneración a lo largo de estos gradientes? Mediante estas preguntas se pretende determinar si la propuesta de cortas de protección debe ser una sola, o si la misma debe adecuarse a las distintas condiciones del paisaje en relación con los gradientes ambientales, aportando el estudio información con base científica a la toma de decisiones en la gestión del recurso forestal nativo. 


\section{MÉTODOS}

Área de estudio. Se trabajó en bosques puros de lenga ubicados en tres zonas geográficas bien diferenciadas donde se realizaron los muestreos (norte, este y sur) donde se desarrolló la cosecha hace 10-12 años (años 20052007), la zona norte correspondió con el ecotono-bosque, la zona sur con el bosque cordillerano, y la zona este con bosques húmedos que alternan con turbales). Para ello se identificaron nueve rodales ( 3 zonas de muestreo x 3 rodales) donde las empresas madereras aplicaron cortas de protección de acuerdo con la normativa vigente en Tierra del Fuego, Argentina (ley provincial 145/94 y normas regulatorias). La superficie total de estudio fue de 684,2 ha de rodales cosechados ubicados en: (i) Ea. Ewan y Lenga Patagonia S.A. en la zona norte $\left(54^{\circ} 22^{\prime} \mathrm{S}, 67^{\circ} 15^{\prime} \mathrm{O}\right)$, (ii) Reserva Forestal de Producción Río Irigoyen en la zona este $\left(54^{\circ} 37^{\prime} \mathrm{S}, 66^{\circ} 39^{\prime} \mathrm{O}\right)$, y (iii) Reserva Forestal de Producción Río Valdez ( $54^{\circ} 38^{\prime} \mathrm{S}, 67^{\circ} 18^{\prime} \mathrm{O}$ ) y Reserva Natural Laguna Negra $\left(54^{\circ} 31^{\prime} \mathrm{S}, 67^{\circ} 18^{\prime} \mathrm{O}\right)$ en la zona sur (figura 1).

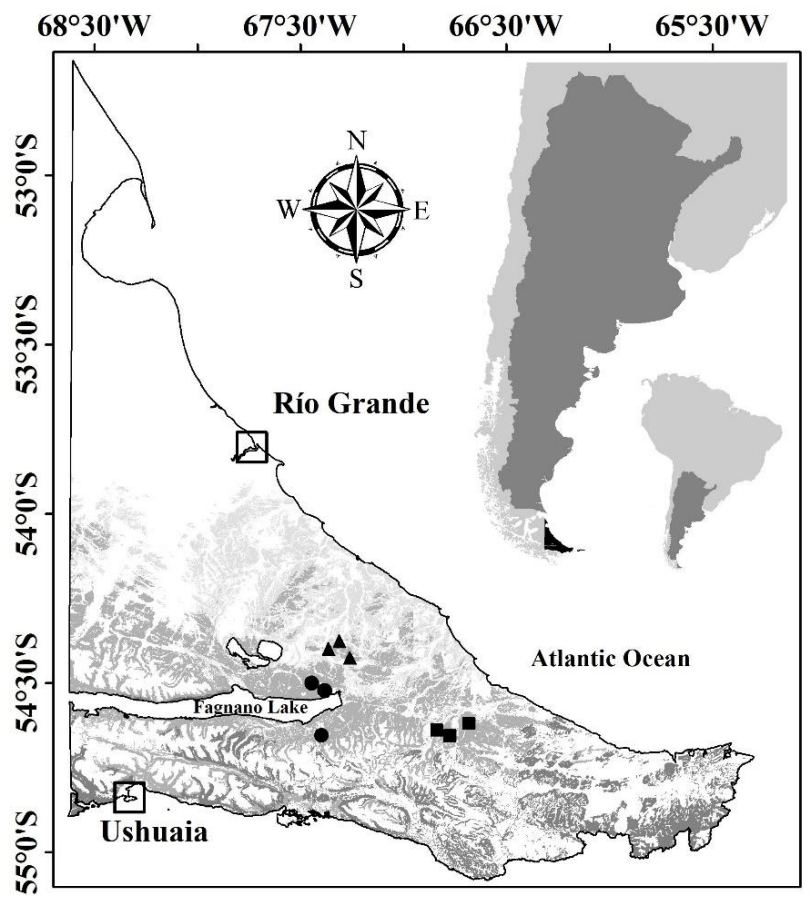

Figura 1. Ubicación de las zonas de muestreo (triángulos en zona norte, cuadrados en zona este, y círculos en la zona sur), y la distribución de los bosques mixtos de Nothofagus betuloides (gris oscuro), puros de $N$. pumilio (gris) y puros de N. antarctica (gris claro).

Location of the sampling sites (triangles in the north area, squares in the east area, and circles in the south area), and the distribution of mixed Nothofagus betuloides (dark grey), pure N. pumilio (grey) and pure $N$. antarctica (pale grey) forests.
Los rodales se vincularon a un sistema de información geográfico (SIG) donde se determinaron las coordenadas del punto central de cada uno. Los rodales seleccionados ( $>10 \mathrm{ha}$ ) se encontraban enmarcados en planes de manejo que fueron fiscalizados por parte de la autoridad de aplicación de Tierra del Fuego, Argentina. Los mismos presentaban una implementación homogénea de la cosecha, y donde su constatación se basó en una caracterización visual (imágenes satelitales SPOT en los dos años posteriores a las intervenciones). Por otra parte, se determinaron las variables climáticas de cada rodal (Hijmans et al. 2005), incluyendo temperatura media anual $\left({ }^{\circ} \mathrm{C}\right)$ (TMA), temperatura media máxima $\left({ }^{\circ} \mathrm{C}\right)(\mathrm{TMAX})$, temperatura media mínima $\left({ }^{\circ} \mathrm{C}\right)(\mathrm{TMIN})$, precipitación media anual $\left(\mathrm{mm}\right.$ año $\left.{ }^{-1}\right)(\mathrm{PA})$, precipitación media del mes más lluvioso $\left(\mathrm{mm} \mathrm{mes} \mathrm{s}^{-1}\right)$ (PMAX), precipitación media del mes más seco $\left(\mathrm{mm} \mathrm{mes}^{-1}\right)$ (PMIN), y evapotranspiración media potencial ( $\mathrm{mm} \mathrm{año}^{-1}$ ) (EVAPO). Los puntos centrales de cada rodal fueron ubicados en el terreno mediante el uso de un geo-posicionador satelital, como punto de partida para establecer cuatro parcelas de muestreo en orientaciones norte, este, sur y oeste (sin corrección de declinación magnética). Cada inicio de parcela de muestreo (transectas de $50 \mathrm{~m}$ x $10 \mathrm{~m}$ cada una) se ubicó a $20 \mathrm{~m}$ del punto central.

Estructura forestal y regeneración. En cada parcela se caracterizaron las siguientes variables para modelar la dinámica de la estructura forestal desde la implementación de la cosecha hasta el presente, siguiendo la metodología propuesta por Gea et al. (2004): (i) diámetro a la altura del pecho (DAP) con corteza $(\mathrm{cm})$ de todos los árboles vivos mayores a $10 \mathrm{~cm}$; (ii) DAP sin corteza $(\mathrm{cm})$ de los árboles muertos en pie y recientemente volteados por viento (ej. presencia de corteza en el fuste) mayores a $10 \mathrm{~cm}$; (ii) diámetro de tocones (DAT) sin corteza $(\mathrm{cm})$ producto de la cosecha; (iii) dos alturas de árboles dominantes (m) para determinar calidad del sitio (CS) (en un sitio 1 los árboles superan los $28 \mathrm{~m}$ y en un sitio 5 no superan los $15 \mathrm{~m}$ de altura a la madurez); y (iv) fases de desarrollo de los árboles vivos como una determinación visual a partir de la corteza. Con estos datos se obtuvieron valores de densidad (N) $\left(\mathrm{n} \mathrm{ha}^{-1}\right)$, área basal $(\mathrm{AB})\left(\mathrm{m}^{2} \mathrm{ha}^{-1}\right)$, volumen total con corteza (VTCC) $\left(\mathrm{m}^{3} \mathrm{ha}^{-1}\right)$ y volumen de trozas sin corteza (VTSC) $\left(\mathrm{m}^{3} \mathrm{ha}^{-1}\right)$. Los detalles de modelos empleados se describen en Martínez Pastur et al. (2002) y Gea et al. (2004).

La regeneración inicial $(<1,3 \mathrm{~m}$ de altura) (parcelas de largo variable x $0,1 \mathrm{~m}$ ) y avanzada $(>1,3 \mathrm{~m}$ de altura $\mathrm{y}<10 \mathrm{~cm}$ DAP) (parcelas de largo variable $\mathrm{x} 1 \mathrm{~m}$ ) se caracterizó mediante dos sub-parcelas de cada tipo ubicadas al comienzo y finalización de cada parcela en cada uno de los rodales (0 y $50 \mathrm{~m}$ ). El tamaño de las parcelas fue variable, donde la longitud se determinó a partir del número de plántulas mensuradas (entre 7 y 10 plantas por parcela). En las parcelas, para cada planta se midió la altura (cm) (A) considerando la longitud existente entre la base y el bro- 
te apical dominante vivo extendiendo el mismo (Martínez Pastur et al. 2011, 2017). También se registró: (i) la calidad forestal (CAL) considerando como plantas adecuadas aquellas sin bifurcaciones, porte recto y buena sanidad (sin presencia de cancros, hongos o hemiparásitas), (ii) daños bióticos debidos al ramoneo de guanaco o ganado doméstico (DB), (iii) daños abióticos (brotes muertos por heladas o sequías) (DA), y (iv) el crecimiento $\left(\mathrm{cm}\right.$ año ${ }^{-1}$ ) en altura de los últimos tres periodos vegetativos (CREC) mediante la identificación de los mucrones. Los daños bióticos por ramoneo se determinaron observando la pérdida de biomasa aérea producida por cortes o remoción y, los daños abióticos cuando se constató muerte de la biomasa aérea de la regeneración (Martínez Pastur et al. 2016, 2017). Por otra parte, con el número de plantas se estimó la densidad de plantas $\left(\right.$ miles ha $\left.^{-1}\right)(\mathrm{N})$ por parcela. Finalmente, la regeneración muestreada se diferenció entre plantas ya establecidas (pre-establecimiento) de aquellas que se instalaron después del aprovechamiento (post-establecimiento), mediante el conteo de mucrones en plantas hasta los 10-12 años (plantas de mayor edad se consideraron como postestablecimiento).

Análisis de los datos. Los valores de estructura forestal fueron empleados para la modelización (Martínez Pastur et al. 2002) de: (i) la estructura original (EO), es decir el estado del bosque primario antes de la cosecha forestal, (ii) la estructura cosechada (EC) que se corresponde con los árboles cortados en el área, (iii) la estructura remanente (ER) que se corresponde a aquella que quedó luego de la cosecha, (iv) la estructura dañada (ED) que consiste en aquellos árboles remanentes post-cosecha que murieron en pie o que fueron afectados por caídas de viento, y (v) la estructura actual (EA) conformada por árboles que se mantuvieron en pie cumpliendo la función de protección al momento de la toma de datos. Para más detalles de estas modelizaciones, ver a Gea et al. (2004). Los valores climáticos, de estructura forestal y regeneración fueron analizados mediante ANOVAs de un factor o múltiples factores considerando una probabilidad del $P=0,05$, donde las diferencias entre medias se compararon mediante el test de Tukey $(P<0,05)$.

\section{RESULTADOS}

Las clases de sitio fueron de mejor calidad en el ecotono (norte) que en la zona este, y donde la zona cordillerana (sur) presentó valores intermedios (alturas dominantes de $23,3 \mathrm{~m}, 22,9 \mathrm{~m}$ y $21,4 \mathrm{~m}$, respectivamente). Las mismas presentaron diferencias significativas entre zonas de muestreo. Las tendencias de temperatura muestran que la zona este presenta valores menores que el resto, donde los valores de evapotranspiración potencial presentan el mismo patrón antes mencionado (norte $>$ sur $>$ este). Sin embargo, ambas variables no llegaron a presentar diferencias significativas entre zonas. La precipitación aumenta desde la zona ecotonal hacia la cordillera y hacia la zona este, encontrándose diferencias significativas entre las distintas zonas de aprovechamiento forestal (cuadro 1).

Las modelizaciones de la estructura forestal variaron de acuerdo con las situaciones analizadas (cuadro 2). La estructura original no presentó diferencias significativas entre las zonas analizadas. Cabe destacar que el área basal en la estructura cosechada fue mayor en la zona este que en la norte y sur presentándose diferencias significativas en el área basal intervenida. Esto influyó en la estructura

Cuadro 1. Análisis de varianza para las diferentes zonas de muestreo (norte, este, sur), analizando clases de sitio y variables climáticas. $\mathrm{CS}=$ clase de sitio (1 alta, 5 baja), TMA = temperatura media anual, TMAX = temperatura media máxima, TMIN = temperatura media mínima, $\mathrm{PA}=$ precipitación media anual, $\mathrm{PMAX}=$ precipitación media del mes más lluvioso, PMIN = precipitación media del mes más seco, EVAPO = evapotranspiración media potencial (ver detalles en Hijmans et al. 2005).

Analyses of variance for the different sampling sites (north, east, south), analyzing site qualities and climatic variables. CS = site quality (1 high, 5 low), TMA = mean annual temperature $\left({ }^{\circ} \mathrm{C}\right), \mathrm{TMAX}=$ maximum mean temperature $\left({ }^{\circ} \mathrm{C}\right), \mathrm{TMIN}=$ minimum mean temperature $\left({ }^{\circ} \mathrm{C}\right), \mathrm{PA}=$ mean annual rainfall $\left(\mathrm{mm} \mathrm{yr}^{-1}\right)$, PMAX $=$ mean rainfall of the wet month $\left(\mathrm{mm} \mathrm{month}^{-1}\right)$, PMIN $=$ mean rainfall of the dry month $\left(\mathrm{mm} \mathrm{month}^{-1}\right)$, EVAPO $=$ mean potential evapotranspiration $\left(\mathrm{mm}_{\mathrm{j}} \mathrm{ear}^{-1}\right)$ (see details in Hijmans et al. 2005).

\begin{tabular}{ccccccccc}
\hline \multirow{2}{*}{ Área } & $\mathrm{CS}$ & TMA & TMAX & TMIN & PA & PMAX & PMIN & EVAPO \\
\cline { 3 - 8 } & & & ${ }^{\circ} \mathrm{C}$ & & $\left(\mathrm{mm} \mathrm{año} \mathrm{O}^{-1}\right)$ & $\left(\mathrm{mm} \mathrm{mes}^{-1}\right)$ & $\left(\mathrm{mm} \mathrm{año}^{-1}\right)$ \\
\hline Este & $3,3 \mathrm{~b}$ & 4,2 & 12,8 & $-3,1$ & $446,3 \mathrm{c}$ & $47,3 \mathrm{c}$ & $25,7 \mathrm{~b}$ & 552,0 \\
Norte & $2,6 \mathrm{a}$ & 4,8 & 13,6 & $-2,3$ & $402,0 \mathrm{a}$ & $42,0 \mathrm{a}$ & $22,7 \mathrm{a}$ & 566,0 \\
Sur & $2,8 \mathrm{ab}$ & 4,9 & 13,6 & $-2,2$ & $425,7 \mathrm{~b}$ & $43,7 \mathrm{~b}$ & $24,7 \mathrm{~b}$ & 563,3 \\
\hline \multirow{2}{*}{$\mathrm{F}(P)$} & 3,35 & 2,43 & 4,28 & 3,63 & 18,20 & 40,20 & 10,50 & 4,28 \\
& $(0,047)$ & $(0,168)$ & $(0,070)$ & $(0,092)$ & $(0,003)$ & $(<0,001)$ & $(0,011)$ & $(0,070)$ \\
\hline
\end{tabular}

$\mathrm{F}=$ prueba de Fisher, $(P)=$ probabilidad. Letras diferentes indican diferencias significativas mediante la prueba de Tukey a 0,05 .

$\mathrm{F}=$ Fisher test,$(P)=$ probability. Different letters showed significant differences using Tukey test at 0.05 . 
remanente: el área basal, volumen total y volumen de trozas que quedó en pie luego de la cosecha resultó mayor en la zona sur que las zonas norte y este, presentándose diferencias significativas para dichas variables. La estructura que sufrió daños luego de la cosecha solo presentó diferencias en el diámetro de los árboles $(\mathrm{F}=0,050)$ pero sin llegar a detectar diferencias entre las medias de las zonas de muestreo analizadas. Finalmente, el área basal de la estructura actual presentó mayores valores en la zona sur que la zona norte y este, donde se encontraron diferencias significativas.
Del análisis de la eficiencia de la cosecha (efectivamente cosechado respecto del potencial del rodal) se observó que la misma fue mayor en el este $(87 \%)$ que en el norte $(83 \%)$ que en el sur (69\%), lo que mayormente explica los valores de estructura remanente y actual. Sin embargo, estas diferencias no llegaron a ser significativas $(\mathrm{F}=1,37, P=0,269)$.

La regeneración pre-instalada fue mayor tanto en densidad como en altura en la zona sur (x2 o x 3 veces que en las zonas este y norte). Los daños bióticos (ramoneo) fueron mayores en la zona norte $(81 \%)$ que en el este

Cuadro 2. Análisis de varianza para las estructuras forestales (original, cosechada, remanentes, dañada y actual) en las diferentes zonas de muestreo (norte, este, sur) comparando la densidad de los árboles (N), diámetro a la altura del pecho (DAP), área basal (AB), volumen total con corteza (VTCC) y volumen de trozas sin corteza (VTSC).

Analyses of variance for the forest structures (original, harvested, remnant, damaged and current) at the different sampling sites (north, east, south), comparing tree density $(\mathrm{N})\left(\mathrm{n} \mathrm{ha}^{-1}\right)$, diameter at breast height $(\mathrm{DAP})(\mathrm{cm})$, basal area $(\mathrm{AB})\left(\mathrm{m}^{2} \mathrm{ha}^{-1}\right)$, total over bark volume $(\mathrm{VTCC})\left(\mathrm{m}^{3}\right.$ $\left.\mathrm{ha}^{-1}\right)$ and timber under bark volume (VTSC) $\left(\mathrm{m}^{3} \mathrm{ha}^{-1}\right)$.

\begin{tabular}{|c|c|c|c|c|c|c|}
\hline Estructura & Área & $\mathrm{N}\left(\mathrm{n} \mathrm{ha}^{-1}\right)$ & $\operatorname{DAP}(\mathrm{cm})$ & $\mathrm{AB}\left(\mathrm{m}^{2} \mathrm{ha}^{-1}\right)$ & $\operatorname{VTCC}\left(\mathrm{m}^{3} \mathrm{ha}^{-1}\right)$ & $\operatorname{VTSC}\left(\mathrm{m}^{3} \mathrm{ha}^{-1}\right)$ \\
\hline \multirow{4}{*}{ Original } & Este & 297 & 44,8 & 51,3 & 524,9 & 324,1 \\
\hline & Norte & 362 & 44,9 & 57,3 & 659,2 & 398,1 \\
\hline & Sur & 387 & 43,6 & 64,2 & 806,6 & 435,0 \\
\hline & $\mathrm{F}(P)$ & $\begin{array}{c}1,35 \\
(0,274)\end{array}$ & $\begin{array}{c}0,06 \\
(0,945)\end{array}$ & $\begin{array}{c}1,34 \\
(0,276)\end{array}$ & $\begin{array}{c}2,41 \\
(0,105)\end{array}$ & $\begin{array}{c}1,82 \\
(0,178)\end{array}$ \\
\hline \multirow{4}{*}{ Cosechada } & Este & 140 & 53,1 & $32,0 \mathrm{~b}$ & 339,6 & 198,1 \\
\hline & Norte & 103 & 50,6 & $20,4 a$ & 235,4 & 142,4 \\
\hline & Sur & 110 & 46,1 & $17,0 \mathrm{a}$ & 192,4 & 118,2 \\
\hline & $\mathrm{F}(P)$ & $\begin{array}{c}0,84 \\
(0,443)\end{array}$ & $\begin{array}{c}1,42 \\
(0,257)\end{array}$ & $\begin{array}{c}5,44 \\
(0,009)\end{array}$ & $\begin{array}{c}2,54 \\
(0,095)\end{array}$ & $\begin{array}{c}2,13 \\
(0,135)\end{array}$ \\
\hline \multirow{4}{*}{ Remanente } & Este & 168 & 40,2 & $22,0 \mathrm{a}$ & $213,6 \mathrm{a}$ & $142,5 \mathrm{a}$ \\
\hline & Norte & 260 & 44,3 & $37,0 \mathrm{ab}$ & $423,8 b$ & $255,5 \mathrm{~b}$ \\
\hline & Sur & 277 & 42,8 & $47,3 b$ & $515,9 \mathrm{~b}$ & $316,8 b$ \\
\hline & $\mathrm{F}(P)$ & $\begin{array}{c}2,58 \\
(0,091)\end{array}$ & $\begin{array}{c}0,30 \\
(0,740)\end{array}$ & $\begin{array}{c}5,86 \\
(0,007)\end{array}$ & $\begin{array}{c}5,34 \\
(0,010)\end{array}$ & $\begin{array}{c}5,17 \\
(0,011)\end{array}$ \\
\hline \multirow{4}{*}{ Dañada } & Este & 133 & 42,3 & 18,9 & 189,9 & 122,8 \\
\hline & Norte & 100 & 54,6 & 19,4 & 230,1 & 133,4 \\
\hline & Sur & 105 & 39,2 & 16,3 & 167,4 & 111,3 \\
\hline & $\mathrm{F}(P)$ & $\begin{array}{c}0,57 \\
(0,571)\end{array}$ & $\begin{array}{c}3,30 \\
(0,050)\end{array}$ & $\begin{array}{c}0,24 \\
(0,787)\end{array}$ & $\begin{array}{c}0,62 \\
(0,544)\end{array}$ & $\begin{array}{c}0,21 \\
(0,808)\end{array}$ \\
\hline \multirow{4}{*}{ Actual } & Este & 70 & 29,8 & $6,2 \mathrm{a}$ & 98,5 & 67,1 \\
\hline & Norte & 185 & 42,5 & $22,3 a b$ & 251,1 & 155,6 \\
\hline & Sur & 187 & 44,3 & $33,8 \mathrm{~b}$ & 380,2 & 224,2 \\
\hline & $\mathrm{F}(P)$ & $\begin{array}{c}1,72 \\
(0,199)\end{array}$ & $\begin{array}{c}2,53 \\
(0,098)\end{array}$ & $\begin{array}{c}5,39 \\
(0,011)\end{array}$ & $\begin{array}{c}3,25 \\
(0,055)\end{array}$ & $\begin{array}{c}3,33 \\
(0,052)\end{array}$ \\
\hline
\end{tabular}

$\mathrm{F}=$ prueba de Fisher, $(P)=$ probabilidad. Letras diferentes indican diferencias significativas mediante la prueba de Tukey a 0,05 .

$\mathrm{F}=$ Fisher test, $(P)=$ probability. Different letters showed significant differences using Tukey test at 0.05 . 
$(36 \%)$ que en el sur $(<1 \%)$. Muchas de estas diferencias no se encontraron para la regeneración post-cosecha, que fue más homogénea a lo largo de las diferentes áreas estudiadas. Sin embargo, los daños bióticos recibidos, que fueron también mayores en la zona norte (29\%) respecto de las otras áreas ( $20 \%$ en el este y $1 \%$ en el sur), generaron un menor desarrollo en altura $(0,2 \mathrm{~m}$ respecto de $0,5-0,6 \mathrm{~m})$, una menor calidad forestal ( $68 \%$ respecto de $79 \%$ - $89 \%$ ) y un menor crecimiento $(2 \mathrm{~cm}$ año-1 respecto de $6-11 \mathrm{~cm}$ año ${ }^{-1}$ ). Si se combinan ambos resultados (regeneración total), que es lo que se evalúa usualmente en la fiscalización de una intervención silvícola, la regeneración fue mayor en el sur $>$ norte $>$ este, sin embargo, la altura promedio fue menor en el norte $(0,3 \mathrm{~m}$ respecto $0,6-1,1 \mathrm{~m})$ debido al ramoneo ( $54 \%$ ) lo que generó menores tasas de crecimiento ( $4 \mathrm{~cm}$ año ${ }^{-1}$ respecto $12-13 \mathrm{~cm}$ año-1 $)$ y menor calidad forestal (55\% respecto de $77-78 \%$ ). Finalmente, la regeneración presentó diferencias significativas a lo largo de las áreas estudiadas (cuadro 3).

\section{DISCUSIÓN}

Las propuestas silvícolas y el paisaje. Las propuestas silvícolas implementadas en los bosques de lenga se han basado en criterios industriales y en base a experiencias efectuadas en otros bosques, principalmente europeos (Gea et al. 2004). Esto ha llevado a la implementación incompleta y/o diferencial de los métodos propuestos a lo largo del paisaje (ej. diferentes empresas poseen diferentes requerimientos de materia prima), siendo necesario un cambio de paradigma en el manejo forestal donde la empresa se adapte a los productos que ofrece cada tipo de bosque. El bosque de lenga no se desarrolla de la misma manera a lo largo del paisaje, siendo influido por los gradientes ambientales naturales que cambian abruptamente a lo largo de Tierra del Fuego (ej. temperatura y precipitación) (Allué et al. 2010, Kreps et al. 2012) (ver cuadro 1). Esto influye también sobre otros componentes del bosque que interactúan en forma natural con la dinámica del bosque, por ejemplo, las poblaciones naturales de guanaco que son mayores en las áreas ecotonales que las cordilleranas (Martínez Pastur et al. 2013, 2016), o el ensamble de especies de plantas del sotobosque que varía a lo largo de todo el paisaje (Pérez Flores et al. 2019, Soler et al. 2019).

Las propuestas silvícolas, en este caso las cortas de protección, se han implementado sin un adecuado estudio de la resiliencia del ecosistema, y sin el monitoreo de la respuesta frente a diferentes conflictos de uso (ej. gana-

Cuadro 3. Análisis de varianza de la regeneración total, pre- y post-instalada respecto de la cosecha en las diferentes zonas de muestreo (norte, este, sur), considerando la densidad (N), altura (A), calidad forestal (CAL), daños abióticos (DA) y bióticos (DB) recibidos, y el crecimiento promedio de las últimas tres temporadas (CREC).

Analyses of variance for the total, pre and post-recruited regeneration considering the harvesting at the different sampling sites (north, east, south), comparing density $(\mathrm{N})$ (thousand ha $\left.{ }^{-1}\right)$, height $(\mathrm{A})(\mathrm{m})$, forest quality $(\mathrm{CAL})(\%)$, abiotic (DA) $(\%)$ and biotic (DB) (\%) damages received, and average growth of the last three seasons (CREC) $\left(\mathrm{cm} \mathrm{yr}^{-1}\right)$.

\begin{tabular}{|c|c|c|c|c|c|c|c|}
\hline TIPO & ÁREA & $\mathrm{N}($ miles ha-1 $)$ & $\mathrm{A}(\mathrm{m})$ & CAL (\%) & DA $(\%)$ & DB (\%) & $\operatorname{CREC}(\mathrm{cm}$ año-1 $)$ \\
\hline \multirow{4}{*}{ Pre- } & Este & $28,7 \mathrm{a}$ & $0,7 \mathrm{a}$ & 75,6 & 3,8 & $36,2 b$ & $15,7 \mathrm{~b}$ \\
\hline & Norte & $34,6 \mathrm{a}$ & $0,5 \mathrm{a}$ & 51,4 & 21,0 & $81,4 \mathrm{c}$ & $6,6 \mathrm{a}$ \\
\hline & Sur & $83,4 \mathrm{~b}$ & $1,5 \mathrm{~b}$ & 67,8 & 4,4 & $0,6 \mathrm{a}$ & $16,0 \mathrm{~b}$ \\
\hline & $\mathrm{F}(P)$ & $\begin{array}{c}3,99 \\
(0,028) \\
\end{array}$ & $\begin{array}{c}11,96 \\
(<0,001)\end{array}$ & $\begin{array}{c}1,90 \\
(0,170)\end{array}$ & $\begin{array}{c}2,89 \\
(0,073)\end{array}$ & $\begin{array}{c}28,84 \\
(<0,001)\end{array}$ & $\begin{array}{c}9,02 \\
(0,001)\end{array}$ \\
\hline \multirow{4}{*}{ Post- } & Este & 50,1 & $0,6 \mathrm{~b}$ & $79,2 \mathrm{ab}$ & 11,6 & $20,3 \mathrm{ab}$ & $10,8 \mathrm{~b}$ \\
\hline & Norte & 68,0 & $0,2 \mathrm{a}$ & $68,3 a$ & 10,4 & $29,3 b$ & $2,0 \mathrm{a}$ \\
\hline & Sur & 101.2 & $0,5 \mathrm{a}$ & $88,5 \mathrm{~b}$ & 5,3 & $1,1 \mathrm{a}$ & $5,8 \mathrm{a}$ \\
\hline & $\mathrm{F}(P)$ & $\begin{array}{c}0,86 \\
(0,433)\end{array}$ & $\begin{array}{c}4,32 \\
(0,023)\end{array}$ & $\begin{array}{c}3,52 \\
(0,042)\end{array}$ & $\begin{array}{c}0,46 \\
(0,639)\end{array}$ & $\begin{array}{c}3,98 \\
(0,029)\end{array}$ & $\begin{array}{c}9,18 \\
(<0,001)\end{array}$ \\
\hline \multirow{4}{*}{ Total } & Este & $78,8 \mathrm{a}$ & $0,6 a$ & $77,8 b$ & 8,5 & $26,7 \mathrm{~b}$ & $12,8 \mathrm{~b}$ \\
\hline & Norte & $102,6 a b$ & $0,3 a$ & $55,2 \mathrm{a}$ & 15,2 & $54,0 \mathrm{c}$ & $4,1 \mathrm{a}$ \\
\hline & Sur & $184,6 \mathrm{c}$ & $1,1 \mathrm{~b}$ & $76,7 \mathrm{~b}$ & 4,8 & $0,8 \mathrm{a}$ & $11,6 \mathrm{~b}$ \\
\hline & $\mathrm{F}(P)$ & $\begin{array}{c}3,26 \\
(0,049)\end{array}$ & $\begin{array}{c}11,38 \\
(<0,001)\end{array}$ & $\begin{array}{c}5,23 \\
(0,008)\end{array}$ & $\begin{array}{c}2,36 \\
(0,104)\end{array}$ & $\begin{array}{c}18,14 \\
(<0,001)\end{array}$ & $\begin{array}{c}12,02 \\
(<0,001)\end{array}$ \\
\hline
\end{tabular}

$\mathrm{F}=$ prueba de Fisher, $(P)=$ probabilidad. Letras diferentes indican diferencias significativas mediante la prueba de Tukey a 0,05 .

$\mathrm{F}=$ Fisher test, $(P)=$ probability. Different letters showed significant differences using Tukey test at 0.05 . 
dería extensiva) y/o amenazas naturales (ej. cambio climático o eventos climáticos extremos) (Martínez Pastur et al. 2013). Tampoco se tuvo en cuenta la topografía (ej. exposiciones o fondos de valle), suelos (ej. humedales o hualves), uso de la tierra (ej. empastamientos debidos a otros usos como la ganadería), tipos de bosque particulares con diferente dinámica (ej. bosques abiertos de baja área basal) o de diferentes estructuras forestales (ej. bosques de estructura irregular). Estas propuestas solo requerían una estructura madura de bosque y la marcación de un área basal remanente en $30 \mathrm{~m}^{2} \mathrm{ha}^{-1}$, lo que derivó en diferencias de respuesta a lo largo del paisaje debido a las consideraciones antes mencionadas.

Influencia de la cosecha y el paisaje sobre la dinámica de la estructura forestal. Los sitios de estudio seleccionados en este trabajo no variaron en su estructura original, siendo de calidad media-alta, que es representativo de las áreas de cosecha en Tierra del Fuego, presentando las mismas características intermedias de las que es posible encontrar a lo largo del paisaje (ej. no se incluyeron exposiciones extremas, ni altitudes extremas, ni rodales con una estructura forestal y/o una dinámica natural particular) (ver cuadro 2, estructura original). Esta selección se realizó para minimizar estos efectos diferenciales que podrían acrecentar las diferencias mencionadas, y poder centrar el análisis en las diferencias que se derivan del paisaje. Bajo estas consideraciones es posible afirmar que la estructura forestal de los bosques naturales sin manejo no varía a lo largo de los gradientes estudiados (pregunta 1). Sin embargo, cabe destacar que se observa una tendencia de los bosques a ser más densos al sur ( $\mathrm{AB}$ $64 \mathrm{~m}^{2} \mathrm{ha}^{-1}$ ) respecto de los del este (AB $\left.51 \mathrm{~m}^{2} \mathrm{ha}^{-1}\right)$ que son más abiertos. Estas diferencias que no llegan a ser significativas, junto con la implementación diferencial de las empresas que aplican el método propuesto generaron diferencias en la intensidad de cosecha (17 a $\left.32 \mathrm{~m}^{2} \mathrm{ha}^{-1}\right)$ $\mathrm{y}$ una consiguiente estructura remanente (ver cuadro 2, estructura remanente). Cabe destacar que el ensamble de especies del sotobosque varía con el paisaje (Pérez Flores et al. 2019, Soler et al. 2019), donde su desarrollo puede generar sinergias positivas o negativas con la regeneración (Martínez Pastur et al. 2013, Silva et al. 2017, Quinteros et al. 2017, Toro Manríquez et al. 2019). Por ejemplo, en la zona este la estructura remanente estuvo por debajo de la normativa de implementación sugerida para las cortas de protección $\left(22 \mathrm{~m}^{2} \mathrm{ha}^{-1}\right)$. En este escenario, es posible afirmar que la intensidad de cosecha implementada no fue igual a lo largo de los gradientes analizados, y que generó estructuras remanentes diferenciales (pregunta 2).

El viento y otros eventos naturales (ej. sequías) modelan la vegetación del paisaje, pudiendo variar significativamente a lo largo del mismo (Rebertus et al. 1997, Martínez Pastur et al. 1999a), donde dichos eventos tienen una influencia diferencial a lo largo de Tierra del Fuego. Por ejemplo, la zona sur recibe una mayor influencia del océa- no Pacífico y la Antártida, mientras que la zona norte está regulada por la influencia del Atlántico sur (Kreps et al. 2012). Es por ello, que hay zonas más propensas a afectaciones por el viento, aunque estas ocurren en mayor o menor magnitud a lo largo de todo el paisaje fueguino (Cellini et al. 2013). En el presente estudio, la estructura forestal dañada durante los 10-12 años posteriores a la cosecha no variaron con las diferentes zonas de estudio (ver cuadro 2 , estructura dañada). Es por ello que se puede afirmar que la dinámica del dosel remanente luego de la cosecha varió en los 10-12 años después de la cosecha a lo largo de los gradientes estudiados (pregunta 3), y que estas diferencias se dieron principalmente por la intensidad de la cosecha y no por los daños ocurridos tras las intervenciones.

Influencia de la cosecha y el paisaje en la regeneración de los rodales cosechados. La regeneración natural de los bosques de lenga representa la etapa más vulnerable del ciclo del manejo, y puede verse afectada por diferentes factores, ej. ciclos de semillas, competencia con el sotobosque, o eventos de sequías o heladas extremas (Rosenfeld et al. 2006, Cellini et al. 2013, Silva et al. 2017, Soler et al. 2019, Toro Manríquez et al. 2019). La lenga requiere de la presencia de un dosel de protección inicial que sirve a un doble propósito, por un lado, como fuente de semillas y, por el otro, de protección frente a condiciones climáticas extremas, limitando a su vez el desarrollo del sotobosque por competencia del agua del suelo y la luz incidente (López Bernal et al. 2003). En este período, la regeneración es semi-tolerante a la sombra. Posteriormente, la regeneración necesita una mayor cantidad de luz y agua, actuando como una especie intolerante a la sombra (Martínez Pastur et al. 2007, 2011, 2013), siendo en esta etapa más vulnerable al ramoneo por parte de diferentes herbívoros nativos y exóticos (Martínez Pastur et al. 2016), donde los mismos ocurren más abundantemente hacia la zona norte en contacto con el ecotono de bosques de ñire (Nothofagus antarctica (G. Forst.) Oerst), y la estepa donde dominan pastizales y arbustales.

La regeneración de un rodal cosechado usualmente comienza a partir de la regeneración pre-instalada proveniente del banco de plántulas del bosque sin intervención, y que es la primera en reaccionar luego de las cortas (Rosenfeld et al. 2006, Martínez Pastur et al. 2017). Esta regeneración es la que dominará el rodal futuro, y se complementa con la regeneración post-cosecha que cubre los sectores sin regeneración pre-instalada. Ambos tipos de regeneración se complementan, y en el caso de la lenga, genera una abundante cantidad de plántulas que al cabo de 15-20 años cubre gran parte del suelo forestal de los bosques cosechados (Rosenfeld et al. 2006, Martínez Pastur et al. 2013). Los bosques analizados presentaron una abundante regeneración $\left(79,102,184\right.$ miles ha $\left.^{-1}\right)$, que estuvo directamente relacionada con el área basal remanente $\left(22,37\right.$ y $\left.47 \mathrm{~m}^{2} \mathrm{ha}^{-1}\right)$ de las áreas este, norte y sur, res- 
pectivamente. Esto ha sido citado en otros trabajos también, donde la regeneración está en relación directa con el canopeo remanente (Martínez Pastur et al. 1999a, 2011, Cellini et al. 2013). La regeneración pre-instalada representó entre el $34 \%$ y el $45 \%$ del total, valores similares a los encontrados en otros estudios (Rosenfeld et al. 2006, Martínez Pastur et al. 2017). A base de estos resultados, se puede afirmar que la respuesta de la regeneración a la cosecha no es homogénea a lo largo de los gradientes estudiados (pregunta 4). Asimismo, como era de esperar se encontró más daño por herbivoría hacia el norte y este, donde se encuentran los establecimientos agropecuarios y ocurren más abundantemente las poblaciones de guanaco (Cavieres et al. 2005, Martínez Pastur et al. 1999b, 2016, 2017), y menos hacia el sur en los sectores cercanos a la cordillera, como también fue descripto en otros estudios (Gea et al. 2004). Sin embargo, no se observaron diferencias por daños abióticos (heladas o sequías), aunque existen diferencias marginalmente significativas $(\mathrm{F}=2,89$, $P=0,073)$ en la regeneración pre-instalada donde la zona norte presenta mayores daños $(21 \%)$ que las zonas este y sur (cercano al $4 \%$ ). Muchas veces, estos daños suelen confundirse con la herbivoría, y suelen ocurrir sinergias negativas (mayor daño abiótico y mayor ramoneo) en años con extremos climáticos (ej. ENSO) (Martínez Pastur et al. 2017). A base de estos resultados se puede decir que efectivamente los daños observados en la regeneración presentan marcadas diferencias a lo largo de las áreas estudiadas (pregunta 5).

Consideraciones a tener en cuenta al implementar propuestas silvícolas a escala de paisaje. La respuesta de los bosques de lenga a escala de paisaje respondió diferencialmente, sin embargo, más allá de las diferencias, todos alcanzaron los estándares mínimos requeridos para la propuesta silvícola de cortas de protección en términos de cantidad de regeneración. Algunas áreas alcanzaron más rápidamente el desarrollo de la regeneración (ej. sur) que permite se apliquen las cortas sucesivas (ej. cortas finales). En otros casos, se observa un retraso producto de un menor crecimiento por factores ambientales o por la presión del ramoneo, tal como fuera citado en otros trabajos (Martínez Pastur et al. 2016, 2017). Sin embargo, no todo se debió a causas naturales o ambientales, sino que algunas respuestas estuvieron relacionadas a la diferente tasa de extracción de las empresas forestales, que influyó directamente en la cantidad de regeneración establecida.

De acuerdo a los interrogantes planteados, se puede determinar que la propuesta de cortas de protección no debe estar basada en una única propuesta, sino que la misma debe adecuarse a las distintas condiciones del paisaje en relación con diferentes gradientes ambientales, y que debe ser controlada para que la misma sea implementada correctamente por las diferentes empresas forestales. Si bien, hay parámetros que no varían con el paisaje (ej. daños posteriores al aprovechamiento o producción de plántulas de acuerdo a una misma área basal remanente), hay otros que sí varían (ej. herbivoría). Es por ello, que se hace necesario establecer programas de monitoreos que definan los umbrales más adecuados de cosecha que no superen la resiliencia de los bosques, y los períodos necesarios para una correcta regeneración de los rodales bajo manejo.

\section{CONCLUSIONES}

A través de esta investigación se intentó brindar nuevas evidencias respecto de la influencia del paisaje en la implementación de un único tratamiento silvícola, que muchas veces es propuesto en una única alternativa de implementación. Las cortas de protección en bosques de lenga presentan diferentes respuestas a lo largo de gradientes ambientales luego de 10 años de realizada la cosecha, donde la dinámica de la estructura forestal remanente y la respuesta de la regeneración natural son buenos indicadores de cambio. En particular se evidencia que, si bien la estructura forestal de los bosques sin manejo no varía significativamente a lo largo de estos gradientes (ej. para una misma calidad de sitio, grado de ocupación y fase de desarrollo), sí se encuentran diferencias debidas a la cosecha implementada por diferentes empresas, principalmente, debido a las ventajas y/o desventajas de la zona en consideración. Esto genera diferentes intensidades de corta que influyeron sobre la dinámica del dosel. Si bien, el daño recibido por la estructura forestal remanente tampoco varió a lo largo de los gradientes estudiados, sí se observan diferencias en la respuesta de la regeneración y su interacción con otros factores (ej. daños por heladas o sequías, así como ramoneo). Basado en estas evidencias se puede inferir la necesidad de implementar diferentes propuestas de cortas de protección, las que deben adecuarse a las distintas condiciones del paisaje en relación con gradientes ambientales que reciban la influencia de diferentes factores bióticos o abióticos.

\section{AGRADECIMIENTOS}

Se desea expresar agradecimientos a profesionales y estudiantes que colaboraron con las tareas de registro de datos en campo y análisis de datos: Tca. Ftal. Hortensia Mansilla, Sra. Vanina Costas, Sr. Jorge Cañas, Sr. Bruno Varela, Tco. Ftal. Juan Andrés Miller y Tco. Gestión Mauricio Giacheti. Al Productor Forestal Luis Mansilla de Aserradero Buenos Aires por proveer de estacas para las parcelas de estudio, al responsable técnico de Lenga Patagonia S.A. Ing. Santiago Favoretti, administradores y propietarios de Ea. Ewan.

\section{REFERENCIAS}

Allué C, J Arranz, J Bava J, J Beneitez, L Collado, J García López. 2010. Caracterización y cartografía fitoclimática del bosque nativo subantártico en la Isla Grande de Tierra del 
Fuego (Patagonia, Argentina). Forest Systems 19(2): 189207. DOI: $10.5424 / \mathrm{fs} / 2010192-01314$.

Cavieres L, A Fajardo. 2005. Browsing by guanaco (Lama guanicoe) on Nothofagus pumilio forest gaps in Tierra del Fuego, Chile. Forest Ecology and Management 204(2-3): 237-248. DOI: 10.1016/j.foreco.2004.09.004.

Cellini JM, G Martínez Pastur, R Soler, M Barrera, MV Lencinas. 2013. Retención variable en bosques de Nothofagus pumilio (Poepp. et Endl.) Krasser en Patagonia Sur: Estructura forestal, estabilidad estructural y regeneración. Yvyraretá 20: 40-47. DOI: no posee.

Gea G, G Martínez Pastur, JM Cellini, MV Lencinas. 2004. Forty years of silvicultural management in southern Nothofagus pumilio (Poepp. et Endl.) Krasser primary forests. Forest Ecology and Management 201: 335-347. DOI: 10.1016/j. foreco.2004.07.015.

Hijmans RJ, SE Cameron, JL Parra, PG Jones, A Jarvis. 2005. Very high-resolution interpolated climate surfaces for global land areas. International Journal of Climatology 25(15): 1965-1978. DOI: 10.1002/joc.1276.

Kreps G, G Martínez Pastur, PL Peri. 2012. Cambio climático en Patagonia Sur: Escenarios futuros en el manejo de los recursos naturales. Ed. Instituto Nacional de Tecnología Agropecuaria, Buenos Aires, Argentina. ISBN 978-987679-137-3.

López Bernal PM, J Bava, SH Antequera. 2003. Regeneración en un bosque de lenga (Nothofagus pumilio (Poepp et Endl.) Krasser) sometido a un manejo de selección en grupos. Bosque 24(2): 13-21. DOI: 10.4067/S071792002003000200002.

Martínez Pastur G, PL Peri, C Fernández, G Staffieri. 1999a. Desarrollo de la regeneración a lo largo del ciclo de manejo forestal de un bosque de Nothofagus pumilio: 1 . Incidencia de la cobertura y el aprovechamiento o cosecha. Bosque 20(2): 39-46. DOI: 10.4206/bosque.1999.v20n2-04.

Martínez Pastur G, PL Peri, C Fernández, G Staffieri, D Rodriguez. 1999b. Desarrollo de la regeneración a lo largo del ciclo del manejo forestal de un bosque de Nothofagus pumilio: 2. Incidencia del ramoneo de Lama guanicoe. Bosque 20(2): 47-53. DOI: 10.4206/bosque.1999.v20n2-05.

Martínez Pastur G, MV Lencinas, JM Cellini, B Díaz, PL Peri, R Vukasovic. 2002. Herramientas disponibles para la construcción de un modelo de producción para la lenga (Nothofagus pumilio) bajo manejo en un gradiente de calidad de sitio. Bosque 23(2): 69-80. DOI: 10.4067/S071792002002000200008 .

Martínez Pastur G, MV Lencinas, PL Peri, M Arena. 2007. Photosynthetic plasticity of Nothofagus pumilio seedlings to light intensity and soil moisture. Forest Ecology and Management 243(2-3): 274-282. DOI: $10.1016 /$ j.foreco.2007.03.034.

Martínez Pastur G, MV Lencinas, JM Cellini, PL Peri, R Soler. 2009. Timber management with variable retention in Nothofagus pumilio forests of Southern Patagonia. Forest Ecology and Management 258(4): 436-443. DOI: 10.1016/j.foreco.2009.01.048.

Martínez Pastur G, PL Peri, JM Cellini, MV Lencinas, M Barrera, H Ivancich. 2011. Canopy structure analysis for estimating forest regeneration dynamics and growth in Nothofagus pumilio forests. Annals of Forest Science 68: 587-594. DOI: $10.1007 / \mathrm{s} 13595-011-0059-1$.
Martínez Pastur G, PL Peri, MV Lencinas, JM Cellini, M Barrera, R Soler, H Ivancich, L Mestre, A Moretto, CB Anderson, F Pulido. 2013. La producción forestal y la conservación de la biodiversidad en los bosques de Nothofagus en Tierra del Fuego y Patagonia Sur. En: Silvicultura en bosques nativos: Avances en la investigación en Chile, Argentina y Nueva Zelanda (P Donoso, A Promis, Eds.). Universidad Austral de Chile, Valdivia, Chile. Cap. 8, 155-179. ISBN: 978-9567173-32.7.

Martínez Pastur G, R Soler, H Ivancich, MV Lencinas, H Bahamonde, PL Peri. 2016. Effectiveness of fencing and hunting to control Lama guanicoe browsing damage: Implications for Nothofagus pumilio regeneration in harvested forests. Journal of Environmental Management 168: 165-174. DOI: 10.1016/j.jenvman.2015.11.051.

Martínez Pastur G, JM Cellini, M Barrera, MV Lencinas, R Soler, PL Peri. 2017. Influencia de factores bióticos y abióticos en el crecimiento de la regeneración pre-y post-cosecha en un bosque de Nothofagus pumilio. Bosque 38(2): 247257. DOI: $10.4067 / \mathrm{S} 0717-92002017000200003$.

Pérez Flores M, G Martínez Pastur, JM Cellini, MV Lencinas. 2019. Recovery of understory assemblage along 50 years after shelterwood cut harvesting in Nothofagus pumilio Southern Patagonian forests. Forest Ecology and Management 450: e117494. DOI: 10.1016/j.foreco.2019.117494.

Quinteros CP, JO Bava, PM López Bernal, M Gobbi, G Defossé. 2017. Competition effects of grazing-modified herbaceous vegetation on growth, survival and water relations of lenga (Nothofagus pumilio) seedlings in a temperate forest of Patagonia, Argentina. Agroforestry Systems 91: 597-611. DOI: $10.1007 / \mathrm{s} 10457-016-9983-2$.

Rebertus AJ, T Kitzberger, T Veblen, LM Roovers. 1997. Blowdown history and landscape patterns in the Andes of Tierra del Fuego, Argentina. Ecology 78(3): 678-692. DOI: $\underline{10.2307 / 2266049}$.

Rosenfeld JM, R Navarro Cerrillo, J Guzmán Álvarez. 2006. Regeneration of Nothofagus pumilio (Poepp. et Endl.) Krasser forests after five years of seed tree cutting. Journal of Environmental Management 78(1): 44-51. DOI: 10.1016/j. jenvman.2005.03.009.

Silva PV, CP Quinteros, AG Greslebin, JO Bava, G Defossé. 2017. Characterization of Nothofagus pumilio (lenga) understory in managed and unmanaged forests of central Patagonia, Argentina. Forest Science 63(2): 173-183. DOI: $\underline{10.5849 / \text { forsci.15-156. }}$.

Soler R, SB Rumpf, S Schindler, G Martínez Pastur, MD Barrera, JM Cellini, M Pérez Flores, F Essl, W Rabitsch, MV Lencinas. 2019. Twelve-year dynamics of alien and native understory plants following variable retention harvesting in Nothofagus pumilio temperate forests in Southern Patagonia. Forest Ecology and Management 449: e117447. DOI: $\underline{\text { 10.1016/j.foreco.2019.07.001. }}$.

Soto DP, DF Jacobs, C Salas, PJ Donoso, C Fuentes, KJ Puettmann. 2017. Light and nitrogen interact to influence regeneration in old-growth Nothofagus-dominated forests in south-central Chile. Forest Ecology and Management 384: 303-313. DOI: 10.1016/j.foreco.2016.11.016.

Soto DP, KJ Puettmann, C Fuentes, DF Jacobs. 2019. Regeneration niches in Nothofagus-dominated old-growth forests after partial disturbance: Insights to overcome arrested succession. Forest Ecology and Management 445: 26-36. DOI: 
10.1016/i.foreco.2019.05.004.

Toro Manríquez MDR, JM Cellini, MV Lencinas, PL Peri, KA

Peña Rojas, G Martínez Pastur. 2019. Suitable conditions for natural regeneration in variable retention harvesting of Southern Patagonian Nothofagus pumilio forests. Ecological Proccesses 8: e18. DOI: 10.1186/s13717-019-0175-7.

Recibido: 06/11/19

Aceptado: 03/03/20 